\title{
A FUNCTIONAL EXPANSION APPROACH TO THE SOLUTION OF NONLINEAR FEEDBACK SYSTEMS*
}

BY

Ram-Nandan P. Singh, Member IEEE and

Timothy L. Johnson, Member IEEE

\section{ABSTRACT}

This paper presents two results: (i) a new structure for the solution of nonlinear analytic systems, and (ii) an application of Bellman's Fundamental Technique to obtain the sub-optimal-feedback control of a class of quasilinear systems with non-quadratic performance indices. The application of the Fundamental Technique with a nonlinear auxiliary equation is shown to result in higher order approximating equations which are linear. The method has been illustrated by three examples.

\footnotetext{
*
} The authors are with the Electronic Systems Laboratory, Department of Electrical Engineering and Computer Science, Massachusetts Institute of Technology, Cambridge, MA 02139. The research has been supported by the National Science Foundation, Grant NSF-76-20860. 


\section{Introduction}

The determination of an optimal feedback control law for a nonlinear system is an important problem in engineering. There are several methods available to attack the problem. The method of dynamic programming yields the feedback solution directly. Unfortunately this method reduces the problem to solving a nonlinear partial differential equation. This is a complex problem, in general. However, the solutions of the feedback problems for linear finite-dimensional systems with a quadratic criterion (known as L-Q problems) are available [1-4].

A more difficult problem than the usual $L-Q$ problem has been mentioned by Anderson and Moore [3, p. 22]. The solutions of the nonlinear partial differential equations resulting from the application of dynamic programming to such problems are unresolved. However, for this particular problem $[3$, p.22], which involves a linear system with a non-quadratic performance index, the exact solution is available [5]. The close examination of this analytical solution reveals the complexity in implementing as well as in deriving the exact feedback laws in general.

In this paper, a method is developed to obtain an asymptotic solution of the feedback laws for more general problems. The problem can be stated as follows:

$$
\text { Find } \begin{aligned}
& U(\cdot) \varepsilon \mathcal{U}:{ }^{j}\left[t_{0}, t_{f}\right] \rightarrow R^{r} \text { to minimize } \\
& J(U(\cdot)): \mathscr{U} \rightarrow R, \\
& J(U)=\int_{t_{0}}^{t_{f}} L(x(t), U(t), t) d t
\end{aligned}
$$


subject to

$$
\dot{x}(t)=\mathscr{F}(x(t), u(t), t) ; x\left(t_{0}\right)=x_{0}
$$

where

$$
x(\cdot):\left[t_{0}, t_{f}\right] \rightarrow R^{n} ; \mathscr{F}: R^{n} \times R^{r} \rightarrow R^{n}
$$

and

$$
L: \quad R^{n} \times R^{r} \rightarrow R_{i} t_{f} \text { is the final time. }
$$

In this paper, we consider the particular class of the above problem [Fig. 1] which is as follows:

(I) The dynamical equation of the system is

$$
\dot{x}(t)=A x(t)+B U(t)+\mathscr{F}_{I}(x(t), U(t))
$$

where

$$
\begin{aligned}
\mathscr{F}_{1}(x, U) & =\psi(x)+\left[\begin{array}{ccc}
U^{T} & N_{1} & f(x) \\
U^{T} & N_{2} & f(x) \\
U^{T} & N_{n} & f(x)
\end{array}\right]+\left[\begin{array}{ccc}
U^{T} & F_{1}(x) & U \\
U^{T} & F_{2}(x) & U \\
U^{T} & F_{n}(x) & U
\end{array}\right] \\
& =\psi(x)+\sum_{i=1}^{n} e_{i} U^{T} N_{i} f(x)+\sum_{i=1}^{n} e_{i} U_{F_{i}}^{T}(x) U
\end{aligned}
$$

where

$$
\psi(x), f(x) \text { are vector polynomials in } x,
$$

and

$$
\begin{aligned}
& F_{i}(x), N_{i} \text { are matrices representing mappings defined as follows: } \\
& F_{i}(\cdot): R^{r} \times R^{n} \rightarrow R^{r}: N_{i}: R^{n} \rightarrow R^{r}
\end{aligned}
$$




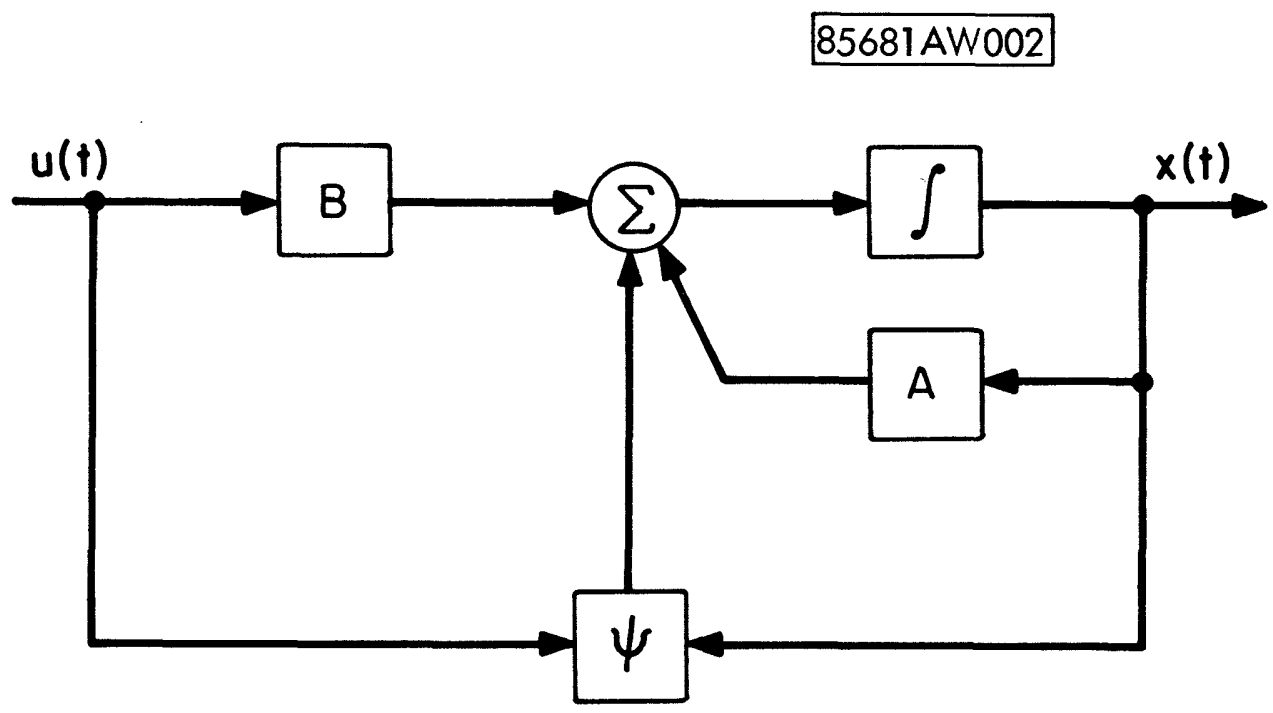

Figure 1(a): Nonlinear plant

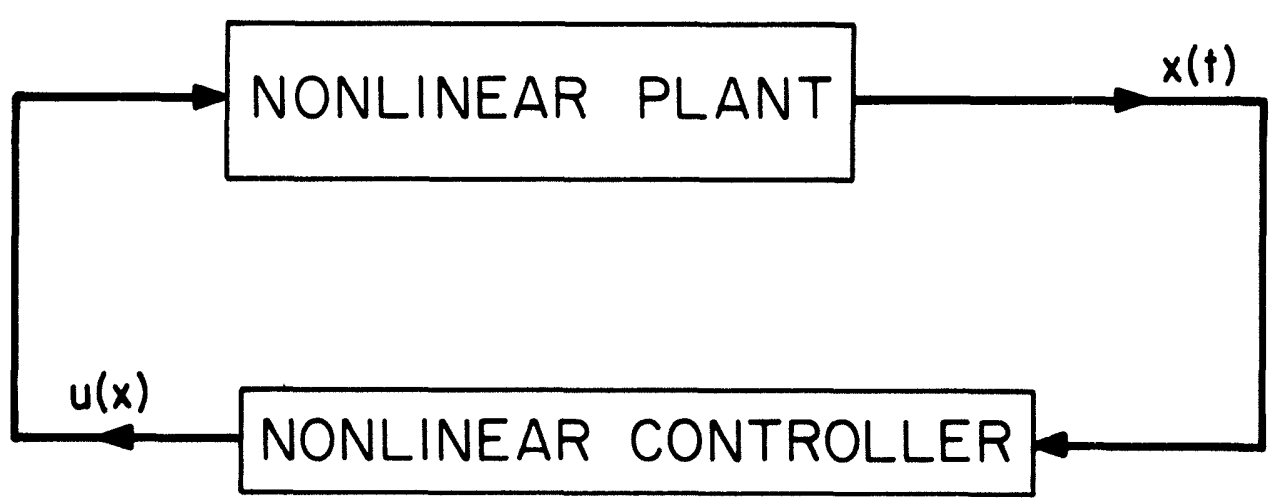

Figure I(b): Closel-loop Nonlinear Plant 
The vectors are defined as

$$
\psi(\cdot): R^{n} \rightarrow R^{n} ; \text { and } f(\cdot): R^{n} \rightarrow R^{n}
$$

A, B represent linear transformations

$$
A: \quad R^{n} \rightarrow R^{n} ; B: R^{r} \rightarrow R^{n} \text {. }
$$

The vectors $e_{i}(i=1,2, \ldots, n)$ are the standard basis vectors.

(II) The scalar functional $L(x, U)$ is defined by

$$
L(x, U) \triangleq U^{T} R U+g(x)
$$

where $g(x)$ is an entire function in $x$ in general, and $R$ is a positive definite $r$ by $r$ matrix. T denotes transpose.

\section{Problem and Detailed Analysis}

Consider a quasilinear dynamical system governed by

$$
\begin{gathered}
\dot{x}=A x+B U+\psi(x)+\sum_{i=1}^{n} e_{i} U^{T} N_{i} f(x)+\sum_{i=1}^{n} e_{i} U^{T} F_{i}(x) U \\
x\left(t_{0}\right)=x_{0}
\end{gathered}
$$

and let the performance index be

$$
J\left[U, t_{0}\right]=\int_{t_{0}}^{t_{f}}\left(U^{T} U+g(x)\right) d t
$$

Find the control function $U(t) \varepsilon \mathscr{U}\left(t_{0} \leq t \leq t_{f}\right)$ such that the cost functional (2) is minimized.

Before proceeding to obtain the solution of the problem, we make the following assumptions: 
Assumption (A): Assume that $\mathscr{H}_{1}(x, u)$ satisfies the following conditions: (i) The nonlinear function $\mathscr{F}_{1}(\mathrm{x}, \mathrm{u})$ is continuous and its first partial derivatives are also continuous.

(ii) $\lim _{|x| \rightarrow 0} \psi(x)=0 ; \lim _{|x| \rightarrow 0} F_{i}(x)=0$ uniformly for $t \varepsilon\left[t_{0}, t_{f}\right]$

(iii) $\lim _{\|(x, u)\| \rightarrow 0} \frac{\| \mathscr{F}_{1}(x, u)||}{\|(x, u)\|}=0$ uniformly for $t \varepsilon\left[t_{0}, t_{f}\right]$

where, $(\mathrm{x}, \mathrm{u}) \varepsilon \mathscr{C}$, and $\mathscr{C}$ denotes the Banach space of continuous functions $(x, u):\left[t_{0}, t_{s}\right] \rightarrow R^{n} \times R^{r}$ with the usual sup norm,

$$
\begin{aligned}
& \|(x, u)\|=\sup _{t}\left\{\sum_{i=1}^{n}\left|x_{i}(t)\right|+\sum_{j=1}^{r}\left|u_{j}(t)\right|: t \varepsilon\left[t_{0}, t_{f}\right]\right\} \\
& \left\|\mathscr{F}_{1}(x, u)\right\| \approx \sup _{t}\left\{\sum_{i=1}^{n}\left|\mathscr{F}_{1 i}(x(t), u(t))\right|\right\}
\end{aligned}
$$

(iv) The linear time-invariant system

$$
\dot{\bar{x}}=A \bar{x}+B \bar{U} ; \bar{x}\left(t_{0}\right)=\bar{x}_{0}
$$

is uniformly completely controllable in the sense of Kalman [7] for bounded coefficients A and B. Here the over-bar denotes variables corresponding to the linear part of system (1) only.

If all the conditions cited above are satisfied by system (1), then the quasilinear system (1) is uniformly controllable [8]. Assumption (B): Assume that $g(x)$ satisfies the following conditions: (i) $g(x)$ is even and positive in $x$, (ii) $g(x)$ is continuous and its first derivative is also continuous and monotonically increasing for positive $\mathrm{x}$. 
(iii) $\lim _{|x| \rightarrow 0} g(x) \rightarrow 0$ uniformly for $t \varepsilon\left[t_{0}, t_{f}\right]$

(iv) $g(x)$ is analytic in $x$.

Now we apply the method of dynamic programming to system (1-2) in order to obtain the optimal feedback law for the system.

The basic Hamilton-Jacobi equation for the above system can be written [3] as

$\frac{\partial V}{\partial t}=-\min _{U(t)}\left\{U^{T} U+\frac{\partial V^{T}}{\partial x}\left[A x+B U+\psi(x)+\sum_{i=1}^{n} e_{i} U^{T} N_{i} f(x)+\sum_{i=1}^{n} e_{i} U^{T} F_{i}(x) U\right]\right\}$

with the terminal condition

$$
V\left(x\left(t_{f}\right), t_{f}\right)=0
$$

where the function $V(x(t), t)$ is assumed to be a function satisfying the conditions on Liapunov's Theorem on asymptotic stability [6], and is twice continuously differentiable.

It can be shown that the minimizing control for equation ( 3 ) is given by

$U^{*}=-\frac{1}{2}\left[I+\frac{1}{2} \sum_{i=1}^{n} \frac{\partial V}{\partial x_{i}}\left[F_{i}^{T}(x)+F_{i}(x)\right]^{-1}\left[B^{T} \frac{\partial V}{\partial x}+\left(\sum_{i=1}^{n} N_{i} \frac{\partial V}{\partial x_{i}}\right) f(x)\right]\right.$

assuming the above inverse exists. Compactly, we can write the above expression as

$$
\mathrm{U}^{*}(\mathrm{x})=\mathscr{G}[\mathrm{V}(\mathrm{x})]
$$

where $\mathscr{G}$ represents the optimal feedback operator. 
Substituting equation (5) into equation (3), we get

$-\frac{\partial V}{\partial t}=\frac{1}{4}\left[\frac{\partial V^{T}}{\partial x} B+\sum_{i=1}^{n} \frac{\partial V}{\partial x_{i}} f^{T}(x) N_{i}^{T}\right]\left[I+\frac{1}{2} \sum_{i=1}^{n} \frac{\partial V}{\partial x_{i}}\left[F_{i}^{T}(x)+F_{i}(x)\right]\right]^{-2}$

$\left[B^{T} \frac{\partial V}{\partial x}+\left(\sum_{i=1}^{n} N_{i} \frac{\partial V}{\partial x_{i}}\right) f(x)\right]+g(x)+\frac{\partial V^{T}}{\partial x} A x$

$-\frac{1}{2} \frac{\partial V^{T}}{\partial x} B\left[I+\frac{1}{2} \sum_{i=1}^{n} \frac{\partial V}{\partial x_{i}}\left[F_{i}^{T}(x)+F_{i}(x)\right]_{1}\right]^{-1}\left[B^{T} \frac{\partial V}{\partial x}+\left(\sum_{i=1}^{n} N_{i} \frac{\partial V}{\partial x_{i}}\right) f(x)\right]$

$+\frac{\partial V^{T}}{\partial x} \psi(x)-\frac{1}{2} \sum_{j=1}^{n} \frac{\partial V}{\partial x_{j}}\left[\frac{\partial V^{T}}{\partial x} B+\sum_{i=1}^{n} \frac{\partial V}{\partial x_{i}} f^{T}(x) N_{i}^{T}\right]\left[I+\frac{1}{2} \sum_{i=1}^{n} \frac{\partial V}{\partial x_{i}}\left[F_{i}(x)+F_{i}(x)\right]\right]^{-1}$

$N_{j} f(x)+\frac{1}{4} \sum_{j=1}^{n} \frac{\partial V}{\partial x_{j}}\left[\frac{\partial V^{T}}{\partial x} B+\sum_{i=1}^{n} \frac{\partial V}{\partial x_{i}} f^{T}(x) N_{i}^{T}\right]\left[I+\frac{1}{2} \sum_{i=1}^{n} \frac{\partial V}{\partial x_{i}}\left[F_{i}^{T}(x)+F_{i}(x)\right]\right]^{-1}$

$F_{j}(x)\left[I+\frac{1}{2} \sum_{i=1}^{n} \frac{\partial V}{x_{i}}\left[F_{i}^{T}(x)+F_{i}(x)\right]\right]^{-1}\left[B^{T} \frac{\partial V}{\partial x}+\left(\sum_{i=1}^{n} N_{i} \frac{\partial V}{\partial x_{i}}\right) f(x)\right]$

With the boundary condition

$$
V\left(x\left(t_{f}\right), t_{f}\right)=0
$$

The results can be summarized as follows:

Theorem 1: Let the quasilinear system (1-2) satisfy assumptions (A) and

(B). Then the optimal feedback control for the system is given by (5)

after solving equation (7).

Obviously equation (7) is a nonlinear first order partial-differential

equation. Under the assumptions (A) and (B), the Cauchy-Kowalewski Theorem [9] guarantees a unique local analytic solution to equation (7). However, 
in general, the solution of equation (7) cannot be obtained in a closed form. Bellman and Bucy [5] have obtained the solution of a problem having a linear system with a non-quadratic performance index. Their results explain the complexity involved in obtaining the solution " $\mathrm{V}(\mathrm{x})$ " of equation (7) analytically.

Since the solution of equation (7) is analytic, the Cauchy-Kowalewski Theorem motivates the application of a regular perturbation expansion of the complete solution to obtain the approximate solution.

Thus, to obtain the optimal feedback solution $U^{*}(x)$, we have to solve equation (7) with the terminal condition (8). In the next section, the Fundamental Technique [10] is applied to obtain the solution. The method, in general, can be applied to systems characterized by (1) and (2) as will be shown in the sequel.

\section{The Fundamental Technique:}

We apply Bellman's Fundamental Technique $[10 ; \mathrm{p} .2]$ to the solution of Hamilton-Jacobi-Bellman equation (7) but using a nonlinear (rather than linear) auxiliary equation. The philosophy behind the Fundamental Technique has been explained by Bellman [10] for the solution of nonlinear problems using a standard equation (called auxiliary equation) which is linear and whose solution thus is well known. The central idea of the Fundamental Technique was thus demonstrated by obtaining an infinite set of linear equations as a solution to the given nonlinear problem.

In this paper, the solution of a nonlinear system is desired using a nonlinear auxiliary equation whose solution is well-known. The method 
yields an infinite set of linear equations as an equivalent solution to the original problem. The salient features of the solution obtained are the following: (i) the first approximating equation is nonlinear and has structure similar to that of the auxiliary equation, (ii) the subsequent approximating equations are linear equations; and (iii) all the equations are recursively connected.

The motivation for extending the application of the Fundamental Technique using a nonlinear auxiliary equation lies in the fact that the form of the nonlinear auxiliary equation used in the technique is closer to the form of the nonlinear problem (which is to be solved) than any form of linear auxiliary equation. Hence this should yield more rapidly convergent approximations to the exact solution.

Further, the results of the linear quadratic theory

(L-Q Theory) can be exploited to achieve this goal.

Now we define some notation which is to be used in the analysis which follows.

Define nonlinear operators $\mathscr{H}$ and $\mathscr{G}$

$$
\mathscr{H}: \quad \mathrm{V} \rightarrow \mathscr{G} \quad \text {; }
$$

where

$$
\begin{aligned}
& \mathrm{V}: \mathrm{R}^{\mathrm{n}} \mathrm{x}\left[\mathrm{t}_{0}, \mathrm{t}_{f}\right] \stackrel{\mathscr{C}}{\rightarrow} \mathrm{R} ; \mathscr{G}: \mathrm{R}^{\mathrm{n} \stackrel{\mathscr{C}}{\rightarrow}} \mathrm{R} \\
& \mathscr{P}: \mathscr{C}^{\infty}\left[\mathrm{R}^{\mathrm{n}} \times\left[\mathrm{t}_{0}, \mathrm{t}_{f}\right]\right] \rightarrow \mathscr{C}^{\infty}\left[\mathrm{R}^{\mathrm{n}}\right]
\end{aligned}
$$

Consider a nonlinear system characterized by

$$
\mathscr{H}[\mathrm{V}]=\mathscr{G}[\mathrm{x}] ; \mathrm{x} \varepsilon \mathrm{R}^{\mathrm{n}}
$$


Assume the nonlinear operator.H is analytic in $\mathrm{V}$ and is thus continuously differentiable with respect to $V(\cdot)$.

The function $V(x)$, we have in mind, is the solution of equation (9). Assume an auxiliary equation with appropriate boundary conditions:

$$
\mathscr{H}_{1}[\mathrm{~V}]=\mathscr{G}_{1}[\mathrm{x}]
$$

whose solution is known. For example, this auxiliary equation may have a form whose solution can be found using a Riccati equation or Burnoulli equation or some other known nonlinear equation. In general, the auxiliary equation may be linear or nonlinear. The application of the Fundamental Technique using a linear auxiliary equation has already been presented [10]. Here we shall deal with a nonlinear auxiliary equation in the Fundamental Technique. In general, the highest degree of nonlinearity present in the auxiliary equation may be the same (if possible) or lower than that of the original nonlinear equation (9).

$$
\begin{aligned}
& \text { Using equation (10), we can rewrite equation (9) as } \\
& \mathscr{H}_{1}[\mathrm{~V}]+\left[\mathscr{H} \cdot[\mathrm{V}]-\mathscr{H}_{1}[\mathrm{~V}]\right]=\mathscr{G}_{1}[\mathrm{x}]+\left[\mathscr{E}[\mathrm{x}]-\mathscr{G}_{1}[\mathrm{x}]\right]
\end{aligned}
$$

or

$$
\mathscr{H}_{1}[\mathrm{~V}]=\mathscr{G}_{1}[\mathrm{x}]+\left[\left(\mathscr{H}_{1}[\mathrm{~V}]-\mathscr{H}[\mathrm{V}]\right)+\left(\mathscr{G}(\mathrm{x})-\mathscr{H}_{1}(\mathrm{x})\right)\right]
$$

or

$$
\mathscr{H}_{1}[\mathrm{~V}]=\mathscr{G}_{1}[\mathrm{x}]+\mathscr{H}_{2}[\mathrm{~V}, \mathrm{x}]
$$

where the expression

$$
\mathscr{H}_{2}[\mathrm{~V}, \mathrm{x}] \triangleq\left[\mathscr{H}_{1}[\mathrm{~V}]-\mathscr{H}[\mathrm{V}]+\mathscr{G}[\mathrm{x}]-\mathscr{G}_{1}[\mathrm{x}]\right]
$$


Introduce a small positive artificial parameter $\varepsilon$ in the above expression. Here $\varepsilon$ is a parameter whose nominal value is one. This is basically a perturbation parameter that can enter the expression (11) in various combinations and powers of $\varepsilon$ as demanded by a particular problem. The placing of $\varepsilon$ in (11) offers high manipulating power to the Fundamental Technique for solving complex nonlinear problems In general, the introduction of this small positive perturbation parameter may be necessary even though the system equation (9) may contain one or more small positive system parameters. However, in special cases, the role of this perturbation parameter may be played by a small positive physical parameter that may enter the system naturally.

For brevity of presentation, let us introduce this artificial parameter in the following form:

$$
\mathscr{H}_{1}[\mathrm{~V}]=\mathscr{G}_{1}[\mathrm{x}]+\varepsilon \mathscr{H}_{2}[\mathrm{~V}, \mathrm{x}]
$$

Thus, this is the required format of the Fundamental Technique.

Since the nonlinear system (11) has been assumed to be analytic in $V(\cdot)$, the solution function can be expressed as

$$
v=v_{0}+\varepsilon v_{1}+\varepsilon^{2} v_{2}+\cdots+\varepsilon^{n} v_{n}+\ldots
$$

where $v_{0}, v_{1}, \ldots, v_{n} \ldots$ are independent of $\varepsilon$. For fixed $v_{0}, v_{1}, v_{2}, \ldots$, the nonlinear operator $\mathscr{H}_{2}[\cdot]$ itself becomes an ordinary function [1I] of $\varepsilon$. Thus

$$
f_{2}(\varepsilon)=\mathscr{H}_{2}[v, x]
$$


At the same time $f_{2}(\varepsilon)$ is also analytic in $\varepsilon$ and hence it can be expanded as a Taylor's series about $\varepsilon=0$. This yields a power series in $\varepsilon$ as given below:

$$
f_{2}(\varepsilon)=\mathscr{H}_{2}\left(\mathrm{v}_{0}+\varepsilon \mathrm{v}_{1}+\varepsilon^{2} \mathrm{v}_{2}+\ldots ; \mathrm{x}\right)=\operatorname{aN}_{0}\left(\mathrm{v}_{0}, \mathrm{x}\right)+\varepsilon \mathscr{N}_{1}\left(\mathrm{v}_{0}, \mathrm{v}_{1} ; \mathrm{x}\right)+\ldots
$$

where $\mathscr{N}_{0}, \mathscr{N}_{1}, \ldots$ are Taylor's series coefficients of the functional $\mathscr{H}_{2}[\mathrm{~V}, \mathrm{x}]$, and can be also obtained by constructing a power series of $\mathscr{H}_{2}(\mathrm{~V}, \mathrm{x})$ in $\varepsilon$.

Now we proceed to obtain an expansion of the functional $\mathscr{H}_{1}[\mathrm{~V}]$ in $\varepsilon$. Since $\mathscr{H}_{1}[\mathrm{~V}]$ is analytic in $\varepsilon[11]$ for fixed $v_{0}, v_{1}, v_{2}, \ldots, v_{n}, \ldots$, this functional becomes an ordinary function of $\varepsilon$. Thus

$$
f_{1}(\varepsilon)=\mathscr{H}_{1}\left[v_{0}+\varepsilon v_{1}+\varepsilon^{2} v_{2}+\ldots\right]
$$

is analytic in $\varepsilon$ about $\varepsilon=0$.

The Taylor's series expantion of a functional has been constructed by Volterra [1];p.24] and he called the expansion an "Extension of Taylor's Theorem" to a class of functionals. Exploiting his ideas, we state a lemma as follows:

Lemma 1:

Assuming the functional is continuous and differentiable on a class of functions over the interval $[a, b]$, Then the Taylor's series expansion of the functional $\mathscr{H}_{1}[V(t)]$ can be expressed as

$$
\mathscr{H}_{1}[\mathrm{~V}]=\mathscr{H}_{1}\left[\mathrm{~V}_{0}\right]+\varepsilon \mathscr{L}_{1}\left[\mathrm{~V}_{1}\right]+\varepsilon^{2} \mathscr{L}_{2}\left[\mathrm{~V}_{2}\right]+\ldots+\varepsilon^{\mathrm{n}} \mathscr{L}_{\mathrm{n}}\left[\mathrm{V}_{\mathrm{n}}\right]+\ldots
$$


,

where

$$
\mathscr{H}_{1}\left[\mathrm{v}_{0}\right], \mathscr{L}_{1}\left[\mathrm{v}_{1}\right], \ldots, \mathscr{L}_{\mathrm{n}}\left[\mathrm{v}_{\mathrm{n}}\right], \ldots
$$

are the Taylor's series coefficients having the following properties:

$\mathscr{H}_{1}\left[\mathrm{~V}_{0}\right]$ is a nonlinear function of $\mathrm{V}_{0} ; \mathscr{P}_{1}\left[\mathrm{~V}_{1}\right]$ is a linear function of $\mathrm{V}_{1}$; $\mathscr{L}_{2}\left[\mathrm{v}_{2}\right]$ is a linear function of $\mathrm{v}_{2}$; and so on.

Proof: We calculate terms of the Taylor's series expansion of a functional as defined by Volterra [11; p.24].

The first term is given by

$$
\left.\mathscr{H}_{I}[\mathrm{~V}]\right|_{\varepsilon=0}=\mathscr{H}_{1}\left[\mathrm{v}_{0}\right]
$$

The second term has been defined as

$$
\left(\frac{\mathrm{d}}{\mathrm{d} \varepsilon} \mathscr{H}_{1}\left[\mathrm{v}_{0}+\varepsilon \phi\right]\right)_{\varepsilon=0}=\left.\int_{\mathrm{a}}^{\mathrm{b}} \mathscr{H}^{\prime}\left[\mathrm{v}_{0}+\varepsilon \phi ; \xi_{1}\right] \frac{\partial \varepsilon \phi\left(\xi_{1}\right)}{\partial \varepsilon} \mathrm{d} \xi_{1}\right|_{\varepsilon=0}
$$

where $\mathscr{H}^{\prime}[\mathrm{V}]$ denotes the first derivative of the functional with respect 1

to $V$ at the point $\xi_{1}$. Here $\xi_{1}$ is a parameter which varies

continuously within $[a, b]$; and in our case,

$$
\phi \triangleq v_{1}+\varepsilon v_{2}+\varepsilon^{2} v_{3}+\cdots
$$

Substituting the value of $\phi$ in the above expression and evaluating at $\varepsilon=0$, we get

$$
\left(\frac{\mathrm{d}}{\mathrm{d} \varepsilon} \mathscr{H}_{1}\left[\mathrm{v}_{0}+\varepsilon \phi\right]\right)_{\varepsilon=0}=\int_{\mathrm{b}}^{\mathrm{a}} \mathscr{P}_{1}^{\prime}\left[\mathrm{v}_{0} ; \xi_{1}\right] \mathrm{v}_{1}\left(\xi_{1}\right) \mathrm{d} \xi_{1}
$$

Clearly for a given $V_{0}$, we write (ii) as a linear function of $v_{1}$. Thus 


$$
\mathscr{L}_{1}\left[\mathrm{v}_{1}\right]=\int_{\mathrm{a}}^{\mathrm{b}} \mathscr{H}_{1}^{\prime}\left[\mathrm{v}_{0} ; \xi_{1}\right] \mathrm{v}_{1}\left(\xi_{1}\right) \mathrm{d} \xi_{1}
$$

To calculate the third term, we have

$$
\begin{aligned}
\left(\frac{\mathrm{d}^{2}}{\mathrm{~d} \varepsilon^{2}} \mathscr{H}_{1}[\mathrm{~V}]\right)_{\varepsilon=0} & =\left(\frac{\mathrm{d}^{2}}{\mathrm{~d} \varepsilon^{2}} \mathscr{H}_{1}\left[\mathrm{v}_{0}+\varepsilon \phi\right]\right)_{\varepsilon=0} \\
& =\left(\frac{\mathrm{d}}{\mathrm{d} \varepsilon}\left(\frac{\mathrm{d}}{\mathrm{d} \varepsilon} \mathscr{H}_{1}\left[\mathrm{v}_{0}+\varepsilon \phi\right]\right)\right)_{\varepsilon=0}
\end{aligned}
$$

Using the expression for the first derivative, we have

$$
\left(\frac{\mathrm{d}^{2}}{\mathrm{~d} \varepsilon^{2}} \mathscr{H}_{1}[\mathrm{~V}]\right)_{\varepsilon=0}=\left(\frac{\mathrm{d}}{\mathrm{d} \varepsilon}\left(\int_{\mathrm{a}}^{\mathrm{b}} \mathscr{H}_{1}^{\prime}\left[\mathrm{v}_{0}+\varepsilon \phi ; \xi_{1}\right] \frac{\partial \varepsilon \phi\left(\xi_{1}\right)}{\partial \varepsilon} \mathrm{d} \xi_{1}\right)\right)_{\varepsilon=0}
$$

Interchanging the order of integration and differentiation (which is permissible), we have

$$
\begin{aligned}
\left(\frac{\mathrm{d}^{2}}{\mathrm{~d} \varepsilon^{2}} \mathscr{H}_{1}[\mathrm{~V}]\right)_{\varepsilon=0}= & \left.\left(\int_{\mathrm{a}}^{\mathrm{b}} \frac{\mathrm{a}}{\mathrm{d} \varepsilon} \mathscr{H}_{1}^{\prime}\left[\mathrm{v}_{0}+\varepsilon \phi ; \xi_{1}\right] \frac{\partial \varepsilon \phi\left(\xi_{1}\right)}{\partial \varepsilon}\right] \mathrm{d} \xi_{1}\right) \\
= & \left(\int_{\mathrm{a}}^{\mathrm{b}} \int_{\mathrm{a}=0}^{\mathrm{b}} \mathscr{H}_{1}^{\prime \prime}\left[\mathrm{v}_{0}+\varepsilon \phi ; \xi_{1}, \xi_{2}\right] \frac{\partial \varepsilon \phi\left(\xi_{2}\right)}{\partial \varepsilon} \mathrm{a} \xi_{2} \frac{\partial \varepsilon \phi\left(\xi_{1}\right)}{\partial \varepsilon} \mathrm{d} \xi_{1}\right) \\
& +\left(\int_{\mathrm{a}}^{\mathrm{b}} \mathscr{H}_{1}^{\prime}\left[\mathrm{v}_{0}+\varepsilon \phi ; \xi_{1}\right] \frac{\mathrm{d}^{2}{ }_{\left(\xi_{1}\right)}}{\mathrm{d}^{2}} \mathrm{~d} \xi_{1}\right)_{\varepsilon=0}
\end{aligned}
$$

Subsituting equation (17) and evaluating as $\varepsilon \rightarrow 0$, we have

$$
\begin{aligned}
\left(\frac{\mathrm{d}^{2}}{\mathrm{~d} \varepsilon^{2}} \mathscr{H}_{1}[\mathrm{v}]\right)_{\varepsilon=0}= & \int_{\mathrm{a}}^{\mathrm{b}} \int_{\mathrm{a}}^{\mathrm{b}} \mathscr{H}_{1}^{\prime \prime}\left[\mathrm{v}_{0} ; \xi_{1}, \xi_{2}\right] \mathrm{v}_{1}\left(\xi_{1}\right) \mathrm{v}_{1}\left(\xi_{2}\right) \mathrm{d} \xi_{,} \mathrm{d} \xi_{2} \\
& +2 ! \int_{\mathrm{a}}^{\mathrm{b}} \mathscr{H}_{1}^{\prime}\left[\mathrm{v}_{0} ; \xi_{1}\right] \mathrm{v}_{2}\left(\xi_{1}\right) \mathrm{d} \xi_{1}
\end{aligned}
$$


For given $v_{0}$ and $v_{1},(i i i)$ is a linear function of $v_{2}$. Hence we can write the third term of the Taylor's series expansion as

$$
\begin{gathered}
\mathscr{L}_{2}\left[\mathrm{v}_{2}\right]=\frac{1}{2 !}\left[\int_{\mathrm{a}}^{\mathrm{b}} \int_{\mathrm{a}}^{\mathrm{b}} \mathscr{f}_{1}^{\mathrm{c}}\left[\mathrm{v}_{0} ; \xi_{1}, \xi_{2}\right] \mathrm{v}_{1}\left(\xi_{1}\right) \mathrm{v}_{1}\left(\xi_{2}\right) \mathrm{d} \xi_{1} \mathrm{~d} \xi_{2}\right. \\
\left.+2 ! \int_{\mathrm{a}}^{\mathrm{b}} e_{1}^{\prime}\left[\mathrm{v}_{0} ; \xi_{1}\right] \mathrm{v}_{2}\left(\xi_{1}\right) \mathrm{d} \xi_{1}\right]
\end{gathered}
$$

Similarly other terms can be calculated. Observe equation (ii) is a nonlinear function of $\mathrm{V}_{0}$ but linear in $\mathrm{V}_{1}$; (iii) is a nonlinear function of $\mathrm{v}_{0}$ and $\mathrm{v}_{1}$, but linear in $\mathrm{v}_{2}$.

Q.E.D.

\section{Theorem 2}

Assume that the auxiliary equation (10) has a known form of solution. The nonlinear system (9) can be imbedded into an infinite set of recursive equations with appropriate boundary conditions:

$$
\begin{aligned}
\mathscr{H}_{1}\left[\mathrm{v}_{0}\right] & =\mathscr{G}_{1}(\mathrm{x}) \\
\mathscr{L}_{1}\left[\mathrm{v}_{1}\right] & =\mathscr{N}_{0}\left[\mathrm{v}_{0} ; \mathrm{x}\right] \\
\mathscr{L}_{2}\left[\mathrm{v}_{2}\right] & =\mathscr{N}_{1}\left[\mathrm{v}_{0}, \mathrm{v}_{1} ; \mathrm{x}\right] \\
& \cdot \cdot \\
\mathscr{L}_{\mathrm{n}}\left[\mathrm{v}_{\mathrm{n}}\right] & =\mathscr{N}_{\mathrm{n}-1}\left[\mathrm{v}_{0}, \mathrm{v}_{1}, \ldots, \mathrm{v}_{\mathrm{n}-1} ; \mathrm{x}\right] \\
& \mathrm{n}=1,2, \ldots \ldots
\end{aligned}
$$

Then the solution of equation (9) can be obtained.

Proof: Using Lemma (1) and equation (14), we can write nonlinear system (9) in its standard format (12) as 


$$
\begin{aligned}
\mathscr{P}_{1}\left[\mathrm{v}_{0}\right]+\varepsilon \mathscr{I}_{1}\left[\mathrm{v}_{1}\right] & +\varepsilon^{2} \mathscr{P}_{[}\left[\mathrm{v}_{2}\right]+\ldots+\varepsilon^{\mathrm{n}} \mathscr{L}_{\mathrm{n}}\left[\mathrm{v}_{\mathrm{n}}\right]+\ldots \\
& =\mathscr{E}_{1}[\mathrm{x}]+\varepsilon\left[\mathscr{N}_{\mathrm{n}}\left(\mathrm{v}_{0} ; \mathrm{x}\right)+\varepsilon \cdot \mathscr{N}_{1}\left(\mathrm{v}_{0}, \mathrm{v}_{1} ; \mathrm{x}\right)+\ldots\right]
\end{aligned}
$$

Equating the coefficients of $\varepsilon^{n}$ from both sides of the above equation, we obtain equation (18).

Clearly the equations (18) are recursively connected. The first nonIinear equation has the same form as that of the auxiliary equation (10). Thus the solution, $v_{0}(x, t)$, of the equation

$$
\mathscr{H}_{I}\left[\mathrm{~V}_{0}\right]=\mathscr{G}_{I}[\mathrm{x}]
$$

is known.

Hence we can obtain the solution of the subsequent linear equations as $v_{1}, v_{2}, \ldots$ Assuming $\varepsilon$ to be an artificial variable with a nominal value one, we can write the solution using equation (13) as

$$
v(x, t)=\sum_{i=1}^{\infty} v_{i}(x, t)
$$

Q.E.D.

Corollary: When the auxiliary equation used in the Fundamental Technique is linear, equations (18) reduce to the following:

$$
\begin{aligned}
\mathscr{L}\left[\mathrm{v}_{0}\right] & =\mathscr{G}_{1}[\mathrm{x}] \\
\mathscr{L}\left[\mathrm{v}_{1}\right] & =\mathscr{V}_{0}\left[\mathrm{v}_{0} ; \mathrm{x}\right] \\
\mathscr{L}\left[\mathrm{v}_{2}\right] & =\mathscr{V}_{1}\left[\mathrm{v}_{0}, \mathrm{v}_{1} ; \mathrm{x}\right] \\
\cdot & \cdot \\
\mathscr{L}\left[\mathrm{v}_{\mathrm{n}}\right] & =\mathscr{N}_{\mathrm{n}-1}\left[\mathrm{v}_{0}, \mathrm{v}_{1}, \ldots, \mathrm{v}_{\mathrm{n}-1} ; \mathrm{x}\right]
\end{aligned}
$$


85681AW001
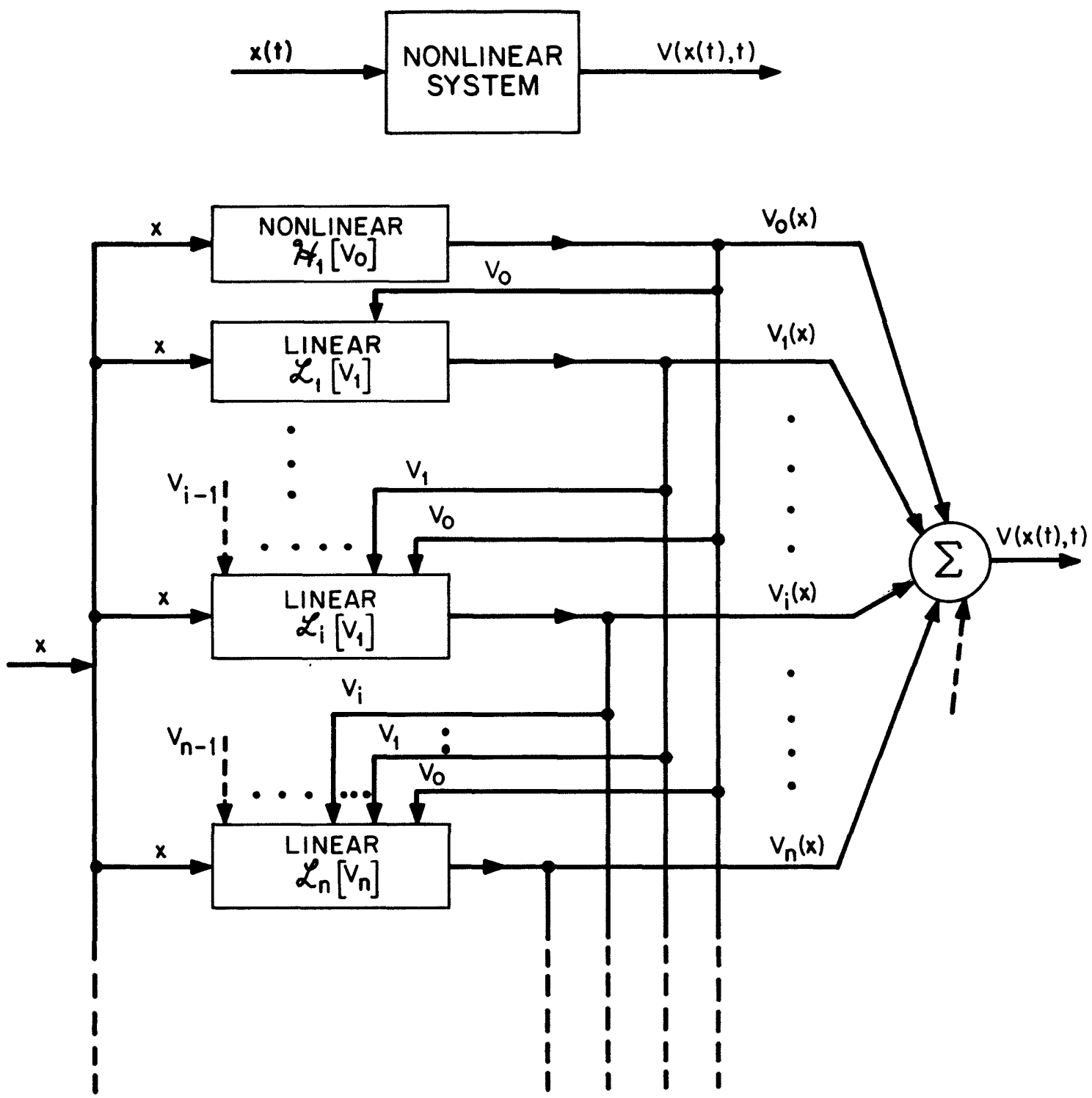

Figure 2: Basic Structure of the Solution of a INonlinear System 
where $\mathscr{L}[\bullet]$ is a linear function which defines the auxiliary equation. The proof of the corollary can easily be obtained using the proof of Lemma 1 and equation (18). Hence it is omitted here.

Remarks:

1. Equation (19) can be obtained independently also as demonstrated by Bellman [10].

2. It may be noted that, in case of a linear auxiliary equation, the functionals $\mathscr{L}_{i}^{[\cdot]}$ in equation (18) become identical as shown in equation (19).

3. Lemma 1 confirms that the coefficients used in equation (14) are functions of their respective arguments only.

Equation (18) represents the basic structure of the solution of a nonlinear system using a nonlinear auxiliary equation in the Fundamental Technique. This has been schematically represented in Figure 2.

The following steps are involved in the method:

1. Choose an auxiliary equation (10).

2. Define a nonlinear functional $\mathscr{H}_{2}[\mathrm{~V}, \mathrm{x}]$ (II).

3. Put the problem in the format (12).

4. Obtain equation (18) starting from equation (12).

5. Solve equation (18) and plug the solutions into equation (13). 
4. The Application of the Method

For brevity of presentation, let $F_{i}(x) \equiv 0$ in equation (1). This

reduces equations (5) and (7) as follows

$$
U^{*}(x)=\left[B^{T} \frac{\partial V}{\partial x}+\sum_{i=1}^{n} N_{i} \frac{\partial V}{\partial x_{i}} f(x)\right]
$$

and

$$
\begin{aligned}
-\frac{\partial V}{\partial t}= & -\frac{1}{4} \frac{\partial V^{T}}{\partial x} B B^{T} \frac{\partial V}{\partial x}+\frac{\partial V^{T}}{\partial x} A x+g(x)+\frac{\partial V^{T}}{\partial x} \psi(x) \\
& +\left[\frac{1}{4}\left(\sum_{i=1}^{n} \frac{\partial V}{\partial x_{i}} f^{T}(x) N_{i}^{T}\right) B^{T} \frac{\partial V}{\partial x}-\frac{3}{4} \frac{\partial V^{T}}{\partial x} B\left(\sum_{i=1}^{n} \frac{\partial V}{\partial x_{i}} N_{i} f(x)\right]\right. \\
& -\frac{1}{4}\left(\sum_{j=1}^{n} \frac{\partial V}{\partial x_{j}}\left(\sum_{i=1}^{n} \frac{\partial V}{\partial x_{i}} f^{T}(x) N_{i}^{T}\right) N_{j} f(x)\right.
\end{aligned}
$$

with $V\left(x\left(t_{f}\right), t_{f}\right)=0$.

From the knowledge of $\mathrm{L}-\mathrm{Q}$ theory, it is well known that

$$
\begin{gathered}
\frac{1}{4} \frac{\partial \vec{V}^{T}}{\partial \bar{x}} \text { B B } \frac{\partial \bar{V}}{\partial x}-\frac{\partial \vec{V}^{T}}{\partial x} A x-\frac{\partial \bar{V}}{\partial t}=x^{T} Q x \\
\text { with } \bar{V}\left(x\left(t_{f}\right), t_{f}\right)=0
\end{gathered}
$$

has a solution of the form

$$
\begin{gathered}
\bar{V}(x(t), t)=x^{T} \bar{k}(t) x \\
\text { with } \bar{k}\left[t_{f}\right]=0
\end{gathered}
$$


Here $Q$ is a positive definite matrix which is known, and $\bar{K}(t)$ is a symmetric matrix satisfying the Riccati matrix equation

$$
\begin{aligned}
\dot{\bar{K}}= & \overline{\mathrm{K}} B B^{T} \bar{K}-\left(\overline{\bar{K}} A+A^{T} \bar{K}\right)-Q \\
& \text { with } \overline{\mathrm{K}}\left[t_{f}\right]=0 .
\end{aligned}
$$

Assuming equation (22) to be an auxiliary equation for the solution of nonlinear equation (21), we can apply the Fundamental Technique to obtain the solution of equation (21).

Now observe that the term $x^{T} Q x$ is not present in equation (21). Hence we shall add and substract this term in equation (21) in order to obtain an equation with the standard format (12). Thus equation (21) can be converted to the form of equation (12) as follows.

$\frac{1}{4} \frac{\partial V^{T}}{\partial x}$ B B ${ }^{T} \frac{\partial V}{\partial x}-\frac{\partial v^{T}}{\partial x} A x-\frac{\partial V}{\partial t}=x^{T} Q x+\varepsilon \cdot \mathscr{H}_{2}(V, x)$

where

$$
\begin{aligned}
e \mathscr{H}_{2}(v, x) \triangleq & g(x)-x^{T} Q \cdot x+\frac{\partial v^{T}}{\partial x} \psi(x)-\frac{1}{4}\left(\sum_{j=1}^{n} \frac{\partial v}{\partial x}\left(\sum_{j=1}^{n} \frac{\partial V}{\partial x_{i}} f^{T}(x) N_{i}^{T}\right) N_{j} f(x)\right) \\
& +\left[\frac{1}{4}\left(\sum_{i=1}^{n} \frac{\partial V}{\partial x_{i}} f^{T}(x) N_{i}^{T}\right) B^{T} \frac{\partial V}{\partial x}-\frac{3}{4} \frac{\partial v^{T}}{\partial x} B\left(\sum_{i=1}^{n} \frac{\partial V}{\partial x_{i}} N_{i} f(x)\right)\right] \\
& \text { and } v\left(x\left(t_{f}\right), t_{f}\right)=0 .
\end{aligned}
$$

Now we can substitute equation (13) in equation (25) and equate the coefficients of various powers of $\varepsilon$. Using equation (18), the following equations are obtained: 


$$
\begin{aligned}
& =\frac{1}{4} \frac{\partial v_{0}^{T}}{\partial x} B B \frac{\partial v_{0}}{\partial x}-\frac{\partial v_{0}^{T}}{\partial x} A x-\frac{\partial v_{0}}{\partial t}=x^{T} Q x \\
& \varepsilon^{1}: \frac{1}{4} \frac{\partial \mathrm{v}_{1}^{\mathrm{T}}}{\partial \mathrm{x}} \mathrm{BB}^{\mathrm{T}} \frac{\partial \mathrm{v}_{0}}{\partial \mathrm{x}}+\frac{1}{4} \frac{\partial \mathrm{v}_{0}^{\mathrm{T}}}{\partial \mathrm{x}} \mathrm{BB}^{\mathrm{T}} \frac{\partial \mathrm{v}_{1}}{\partial \mathrm{x}}-\frac{\partial \mathrm{v}_{1}^{\mathrm{T}}}{\partial \mathrm{x}} \mathrm{Ax}-\frac{\partial \mathrm{v}_{1}}{\partial t}=\mathrm{N}_{0}\left(\mathrm{v}_{0} ; \mathrm{x}\right) \\
& \varepsilon^{2}: \frac{1}{4} \frac{\partial v_{2}^{T}}{\partial x} B B^{T} \frac{\partial v_{0}}{\partial x}+\frac{1}{4} \frac{\partial v_{0}^{T}}{\partial x} B B^{T} \frac{\partial v_{2}}{\partial x}+\frac{1}{4} \frac{\partial v_{1}^{T}}{\partial x} B B^{T} \frac{\partial v_{1}}{\partial x} \\
& -\frac{\partial v_{2}^{T}}{\partial x} A X-\frac{\partial v_{2}}{\partial t}=N_{1}\left(v_{0}, v_{i} ; x\right) \\
& \varepsilon^{n}: \frac{1}{4} \sum_{i=0}^{n}\left(\frac{\partial v_{n-i}^{T}}{\partial x} B B^{T} \frac{\partial v_{i}}{\partial x}\right),-\frac{\partial v_{n}^{T}}{\partial x} A x-\frac{\partial v_{n}}{\partial t}=N_{n-1}\left(v_{0}, v_{1}, \ldots, v_{n-1} ; x\right) \\
& n=1,2, \ldots .
\end{aligned}
$$

(i) Observe that the first equation in (26) is a nonlinear (quadratic) first-order partial differential equation in $V_{0}(t)$ and has a well-known solution as given by equation (23). The other subsequent equations are linear first order partial differential equations in $v_{1}, v_{2}, \ldots$ respectively.

(ii) They are recursively connected and the $n^{\text {th }}$ equation contains all the solutions of the previous $(n-1)$ equations.

The above result can be summarized as follows:

Theorem 3: Let $F_{i}(t) \equiv 0$ for all $x \varepsilon R^{n}$, and system (1) and (2) satisfy the assumptions (A) and (B). Then the solution of equation (21) can be used to construct the optimal feedback control as

$$
U^{*}(x)=\left[B^{T} \frac{\partial V}{\partial x}+\sum_{i=1}^{n} N_{i} \frac{\partial V}{\partial x_{i}} f(x)\right]
$$


Using equation (6), we can write equation (22) as

$$
u^{*}(x(t), t)=\mathscr{G}[V(x(t), t)]
$$

The schematic representation of the open-loop and closed loop nonlinear controllers are given in Figure 2 and Figure 3 respectively. Figure 3 shows that the nonlinear controller has been imbedded into a hierarchy of subcontrollers.

\section{Example 1}

Consider a nonlinear system

$$
\dot{x}=x+\mu x^{3}+u ; x(0)=0
$$

with the cost functional

$$
J[u]=\int_{0}^{t} f\left(u^{2}+x^{2}+\frac{1}{2} x^{4}\right) d t
$$

to be minimized.

Here $\mu$ is a parameter of the system that has entered the governing equation naturally.

Clearly the system satisfies the assumptions (A) and (B). The Hamilton-Jacobi equation for the above problem is given by

$$
\frac{\partial V}{\partial t}=-\min _{u^{*}}\left\{u^{2}+x^{2}+\frac{1}{2} x^{4}+\frac{\partial V}{\partial x}\left(u+x+\mu x^{3}\right)\right\}
$$

The minimizing control is found using the formula (5) as

$$
u^{*}=-\frac{1}{2} \frac{\partial V}{\partial x}
$$

Substituting this in the above equation, we have

$$
\frac{\partial V}{\partial t}=\frac{1}{4}\left(\frac{\partial V}{\partial x}\right)^{2}-x^{2}-\mu \frac{\partial V}{\partial x} x^{3}-\frac{1}{4} x^{4}-\frac{\partial V}{\partial x}
$$


with the terminal condition $V\left(x\left(t_{f}\right), t_{f}\right)=0$.

This is a nonlinear first order partial differential equation and corresponds to equation (7) of section 2 .

In order to apply the Fundamental Technique we have to choose a suitable auxiliary equation. Let

$$
\frac{1}{4}\left(\frac{\partial v}{\partial x}\right)^{2}-\frac{\partial v}{\partial x} x-\frac{\partial v}{\partial t}=x^{2}
$$

be the nonlinear auxiliary equation (22). Define

$$
\mathscr{H}_{2}[\mathrm{v}, \mathrm{x}] \triangleq \mu \frac{\partial \mathrm{v}}{\partial \mathrm{x}} \quad \mathrm{x}^{3}+\frac{1}{4} \quad \mathrm{x}^{4}
$$

Now we can put equation (29) into a standard format (12) as required by the Technique

$$
\frac{1}{4}\left(\frac{\partial V}{\partial x}\right)^{2}-\frac{\partial V}{\partial x} x-\frac{\partial V}{\partial t}=x^{2}+\varepsilon \mathscr{H}[V, x] ; V\left(x\left(t_{f}\right), t_{f}\right)=0
$$

It may be observed that if $\mathscr{H} \mathscr{H}_{2}[\mathrm{~V}, \mathrm{x}]$ is expanded in terms of $\mu$, the first approximation of equation (18) cannot be obtained. This must be of the same form as that of the above auxiliary equation. This is essential for the method to. work. Hence the straight-forward perturbation expansion substitutions [12-16] do not work. Thus the introduction of $\varepsilon$ is essential.

Following the procedures as outlined in Section 3, we obtain equation

(18) as follows:

$$
\begin{aligned}
& \varepsilon^{0}: \frac{1}{4}\left(\frac{\partial v_{0}}{\partial x}\right)^{2}-\left(\frac{\partial v_{0}}{\partial x}\right) x-\frac{\partial v_{0}}{\partial x}=x^{2} ; \\
& \text { with the terminal condition } v_{0}\left(x\left(t_{f}\right), t_{f}\right)=0
\end{aligned}
$$


$\varepsilon^{1}: \frac{1}{2} \frac{\partial \mathrm{V}_{1}}{\partial \mathrm{x}} \frac{\partial \mathrm{v}_{0}}{\partial \mathrm{x}}-\frac{\partial \mathrm{V}_{1}}{\partial \mathrm{x}} \mathrm{x}-\frac{\partial \mathrm{V}_{1}}{\partial t}=\frac{\mathrm{x}^{4}}{4} ;$

with terminal condition $v_{1}\left(x\left(t_{f}\right), t_{f}\right)=0$

$\varepsilon^{2}: \frac{1}{2} \frac{\partial v_{2}}{\partial x} \frac{\partial v_{0}}{\partial x}+\frac{1}{4}\left(\frac{\partial v_{1}}{\partial x}\right)^{2}-\frac{\partial v_{2}}{\partial x} x-\frac{\partial v_{2}}{\partial t}=\mu \frac{\partial v_{0}}{\partial x} x^{3} ;$

with the terminal condition $v_{2}\left(x\left(t_{f}\right), t_{f}\right)=0$

$$
\begin{array}{cc}
\varepsilon^{n}: \frac{1}{4} \sum_{i=0}^{n} \frac{\partial v_{n-i}}{\partial x} \frac{\partial v_{i}}{\partial x}-\frac{\partial v_{n}}{\partial x} x-\frac{\partial v_{n}}{\partial t}=\mu \frac{\partial v_{n-2}}{\partial x} x^{3} ; \\
(n=2,3, \ldots) & v_{n}\left(x\left(t_{f}\right), t_{f}\right)=0
\end{array}
$$

The first equation is a nonlinear equation whose solution is given by

$$
v_{0}(x(t), t)=k(t) x^{2}
$$

where the gain $k(t)$ is the solution of the following Riccati equation

$$
\frac{d k}{d t}+2 k-k^{2}+1=0 ; k\left(t_{f}\right)=0
$$

The higher order approximations $\mathrm{v}_{1}(\mathrm{x}), \mathrm{v}_{2}(\mathrm{x}), \ldots$ can be obtained after solving the subsequent linear equations.

Thus the sub-optimal feedback control is given by the finite terms and putting $\varepsilon=1$,

$$
u^{*}(x, t)=-\frac{1}{2} \sum_{i=1}^{n} \frac{\partial v_{i}}{\partial x} \triangleq \quad \mathscr{G}[v(x, t)]
$$

where the optimal feedback operator

$$
\mathscr{G}[\cdot] \triangleq-\frac{1}{2} \sum \frac{\partial}{\partial x}[\cdot]
$$

The structure of the sub-optimal feedback controller is similar to that given in Figure 3 . 


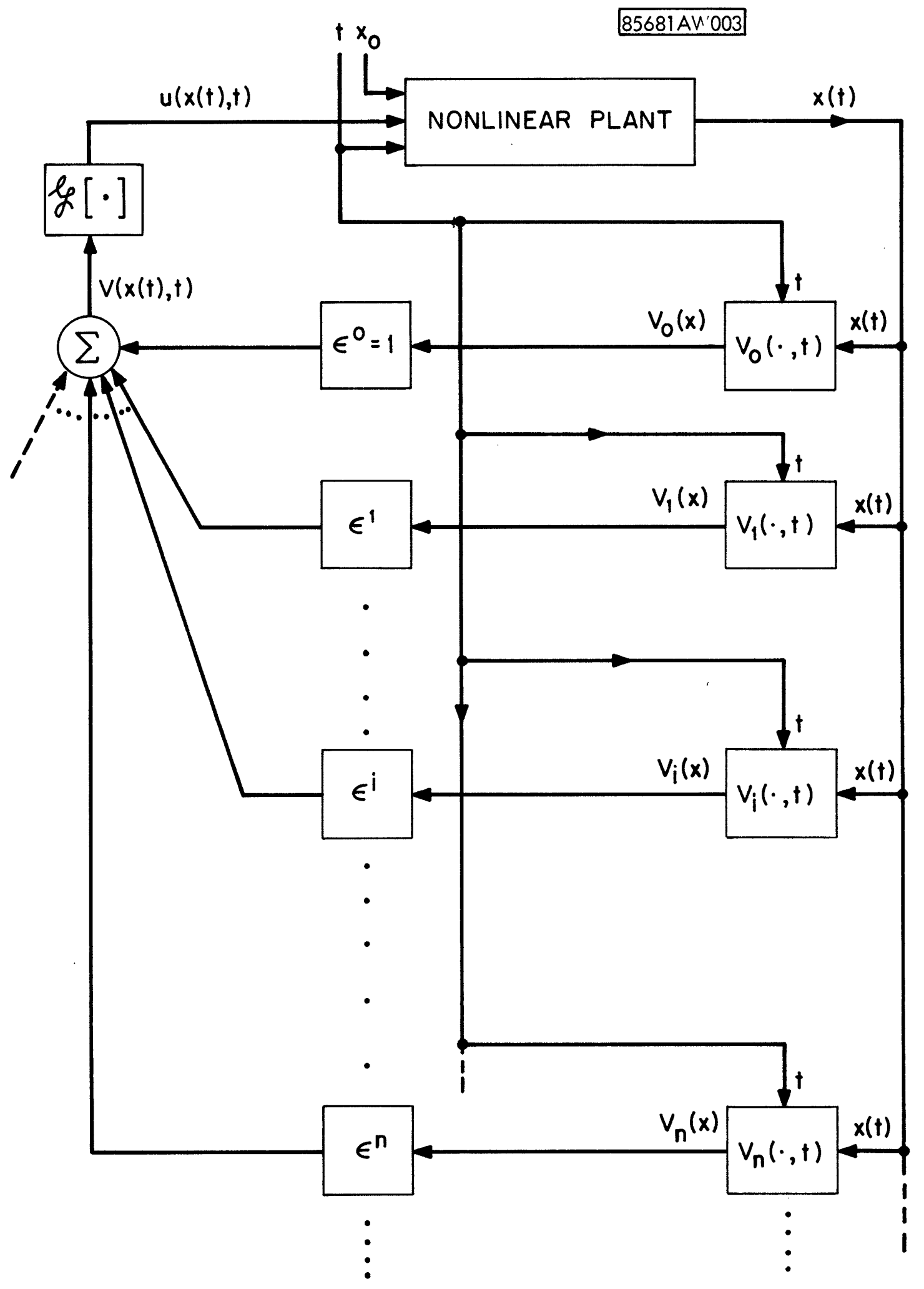

Figure 3

Basic Structure of the Non-Linear Controller 
Example 2:

Consider a nonlinear system characterized by

$\dot{x}=a x+b u+c x u ;$

with the initial condition $\mathrm{x}(0)=\mathrm{x}_{0}$

and the performance index to be minimized is

$\int_{0}^{t} f\left(u^{2}+x^{2}+x^{6}\right) d t$

The above equations satisfy the assumptions (A) and (B) of Section 2 .

Following the procedures we obtain equation (5) and (7) as

$$
u *(x)=-\left(\frac{b+c x}{2}\right) \frac{\partial v}{\partial x}
$$

and

$$
\frac{\partial V}{\partial t}=\frac{1}{4}(b+c x)\left(\frac{\partial V}{\partial x}\right)^{2}-x^{2}-x^{6}-a \frac{\partial V}{\partial x} x ;
$$

with the terminal condition $V\left(x\left(t_{f}\right), t_{f}\right)=0$

choose an auxiliary equation

$$
\frac{b}{4}\left(\frac{\partial V}{\partial x}\right)^{2}-\frac{\partial V}{\partial x} \text { ax }-\frac{\partial V}{\partial t}=x^{2}
$$

Define the nonlinear operator

$$
\mathscr{P}_{2}[\mathrm{v}, \mathrm{x}] \triangleq\left[\frac{\mathrm{Cx}}{4}\left(\frac{\partial \mathrm{v}}{\partial \mathrm{x}}\right)^{2}+\mathrm{x}^{6}\right]
$$

using these, we have the standard format (12) as

$$
\frac{b}{4}\left(\frac{\partial v}{\partial x}\right)^{2}-\frac{\partial v}{\partial x} a x-\frac{\partial v}{\partial t}=x^{2}+\varepsilon \mathscr{H}_{2}(v, x)
$$

with the terminal condition $V\left(x\left(t_{f}\right), t_{f}\right)=0$ 
obtain equation (18) as

$\varepsilon^{0}: \frac{b}{4}\left(\frac{\partial v_{0}}{\partial x}\right)^{2}-\frac{\partial v_{0}}{\partial x}$ ax $-\frac{\partial v_{0}}{\partial t}=x^{2}$

with the terminal condition $v_{0}\left(x\left(t_{f}\right), t_{f}\right)=0$.

$\varepsilon^{1}: \frac{b}{4}\left(\frac{\partial v_{0}}{\partial x} \frac{\partial v_{1}}{\partial x}\right)-\frac{\partial v_{1}}{\partial x}$ ax $-\frac{\partial v_{1}}{\partial t}=\frac{c}{4} x\left(\frac{\partial v_{0}}{\partial x}\right)^{2}+x^{6} ;$

terminal condition $v_{I}\left(x\left(t_{f}\right), t_{f}\right)=0$.

${ }_{\varepsilon}^{n}: \frac{b}{4} \sum_{i=1}^{n} \frac{\partial v_{n-i}}{\partial x} \frac{\partial v_{i}}{\partial x}-\frac{\partial v_{n}}{\partial x} a x-\frac{\partial v_{n}}{\partial t}=\frac{c}{4} x \sum_{i=0}^{n-1} \frac{\partial v_{n-i-1}}{\partial x} \frac{\partial v_{i}}{\partial x} ;$

$(n=2,3, \ldots)$

$$
v_{n}\left(x\left(t_{f}\right), t_{f}\right)=0
$$

Clearly the first equation is nonlinear and whose solution is given by

$\mathrm{v}_{0}=\mathrm{k} \mathrm{x}^{2}$

where $\mathrm{k}$ is the solution of the Riccati equation

$\frac{d k}{d t}+2 a k-\frac{b}{4} k^{2}+1=0 ; k\left(t_{f}\right)=0$.

The higher order approximations $v_{1}, v_{2}, \ldots$ can be obtained after solving the first order linear partial-differential equations sequentially.

Putting $\varepsilon=1$, we obtain the approximate solution as

$u^{*}(x, t)=-\left(\frac{b+c x}{2}\right) \sum_{n=0}^{N} \frac{\partial v_{n}}{\partial x}$

This can also be written as 


$$
u^{*}(\mathrm{x}, \mathrm{t})=\mathscr{G}[\mathrm{v}(\mathrm{x}, \mathrm{t})],
$$

where the optimal feedback operator

$$
\mathscr{G}[\cdot] \triangleq-\left(\frac{\mathrm{b}+\mathrm{cx}}{2}\right) \sum \frac{\partial}{\partial \mathrm{x}}[\cdot]
$$

the structure is similar to that depicted in Figure 3.

Example 3: [Garrard 13]

A controllable dynamic system is given by

$$
\dot{x}=A x+\mu \psi(x)+B u ; x(0)=x_{0}
$$

and the cost functional is

$$
J=\frac{1}{2} \int_{0}^{\infty} \quad\left(x^{\prime} Q x+u^{\prime} R u\right) d t
$$

The problem is to determine the optimal feedback control $u *(x, t)$

such that the system is transferred from its initial $x_{0}$ to the origin while minimizing the cost functional. There is no bound on the control function.

The minimizing control $u *(x, t)$ can be calculated as

$$
\mathrm{u}^{*}=-\mathrm{R}^{-1} \mathrm{~B}^{\mathrm{T}} \frac{\partial \mathrm{V}}{\partial \mathrm{x}} ; \mathrm{x}(\infty)=0
$$

Then the equation (7) reduces to

$$
\frac{1}{2} \frac{\partial V^{T}}{\partial x} B R^{-1} B^{T} \frac{\partial V}{\partial x}-\frac{\partial V^{T}}{\partial x} A x=\frac{1}{2} x^{T} Q x+\mu \frac{\partial V^{T}}{\partial x} \psi(x) ;
$$

the terminal condition $\mathrm{V}(\mathrm{x}(\infty), \infty)=0$ 
Following the steps of Bellman's Fundamental Technique, we proceed as follows:

(i) Choose a nonlinear auxiliary equation

$$
\begin{aligned}
\frac{1}{2} \frac{\partial \overrightarrow{\mathrm{v}}^{T}}{\partial \mathrm{x}} \mathrm{BR}^{-1} \mathrm{~B}^{\mathrm{T}} \frac{\partial \overline{\mathrm{v}}}{\partial \mathrm{x}}-\frac{\partial \overline{\mathrm{V}}}{\partial \mathrm{x}} \mathrm{Ax}=\frac{1}{2} \mathrm{x}^{\mathrm{T}} 2 \mathrm{x} ; \\
\overline{\mathrm{V}}(0, \infty)=0
\end{aligned}
$$

whose solution can be expressed as

$$
\overline{\mathrm{V}}=\mathrm{x}^{\mathrm{T}} \overline{\mathrm{k}} \mathrm{x}
$$

where $\bar{k}$ is a symmetric positive definite matrix and is the solution of the matrix Riccati equation

$$
\overline{k A}+A^{T} \bar{k}-\overline{k B R}^{-1} \mathrm{~B}_{\bar{k}}+Q=0
$$

(ii) Define a nonlinear functional

$$
\mathscr{H}_{2}(v, x) \triangleq \mu \frac{\partial v^{T}}{\partial x} \psi(x)
$$

(iii) Write the equation in the required format as

$$
\frac{1}{2} \frac{\partial v^{T}}{\partial x} B R^{-1} B^{T} \frac{\partial V}{\partial x}-\frac{\partial v^{T}}{\partial x} A x=\frac{1}{2} x^{T} 2 x+\varepsilon \cot (V ; x)
$$

(iv) We obtain equation (18) as

$$
\begin{aligned}
& \mu^{0}: \frac{1}{2} \frac{\partial v_{0}^{T}}{\partial x} B R^{T}{ }^{T} \frac{\partial v_{0}}{\partial x}-\frac{\partial v_{0}^{T}}{\partial x} A x=\frac{1}{2} x^{T} Q x ; \\
& \text { the terminal condition is } v_{0}(0, \infty)=0
\end{aligned}
$$




$$
\begin{gathered}
\mu^{1}: \frac{\partial v_{1}^{T}}{\partial x} B R^{-1}{ }_{B}^{T} \frac{\partial v_{0}}{\partial x}+\frac{\partial v_{0}^{T}}{\partial x} B R^{-1}{ }_{B}^{T} \frac{\partial v_{1}}{\partial x}-\frac{\partial v_{1}^{T}}{\partial x} A x=\mu \frac{\partial v_{0}^{T}}{\partial x} \psi(x) ; \\
V_{1}(0, \infty)=0 .
\end{gathered}
$$

$\mu=\frac{1}{2} \sum_{i=1}^{n}\left(\frac{\partial v_{n-i}^{T}}{\partial x} B^{-1}{ }_{B}^{T} \frac{\partial v_{i}}{\partial x}\right)-\frac{\partial v_{n}^{T}}{\partial x} A x=\mu \frac{\partial v_{n-1}^{T}}{\partial x} \psi(x)$ the terminal condition $v_{n}(0, \infty)=0$

(v) Since the solution of the first equation is known, the subsequent equations are first order linear partial differential equations and recursively connected. Hence using the method of characteristics, the solutions $v_{1}, v_{2}, \ldots$ can be obtained.

Thus the optimal control is given as

$$
u^{*}(x)=-R^{-1} B^{T} \sum_{n=1}^{\infty} \frac{\partial v_{n}}{\partial x}
$$

The structure of this feedback control has been shown in Figure 3 . Here

$$
\mathrm{u}^{*}(\mathrm{x})=\mathscr{G}[\mathrm{v}(\mathrm{x})]
$$

where the optimal feedback operation is

$$
\mathscr{G}[\cdot] \triangleq-\mathrm{R}^{-1}{ }_{\mathrm{B}}^{\mathrm{T}} \cdot \sum \frac{\partial}{\partial \mathrm{x}} \cdot[\cdot]
$$

Note: In this particular problem, the system parameter $\mu$ may also replace the role of $\varepsilon$. Thus we modify the expressions as follows.

$$
\bar{v} \triangleq \bar{v}_{0}+\mu \bar{v}_{1}+\mu^{2} \bar{v}_{2}+\cdots
$$


The nonlinear functional

$$
\overline{\mathscr{H}}_{2}(\overline{\mathrm{V}}, \mathrm{x}) \triangleq \frac{\partial \overrightarrow{\mathrm{V}}^{\mathrm{T}}}{\partial \mathrm{x}} \psi(\mathrm{x}) ;
$$

The required format becomes

$$
\frac{1}{2} \frac{\partial \vec{V}^{T}}{\partial x} B R^{-1} B^{T} \frac{\partial \bar{V}}{\partial x}-\frac{\partial \vec{V}^{T}}{\partial x} A x=\frac{1}{2} x^{T} Q x+\sqrt{\mathscr{Y} /}(\bar{V}, x) ;
$$

with the terminal condition $\bar{v}(0, \infty)=0$. (Overbar indicates the corresponding variables.)

Following steps (iv) and (v), the solution can be expressed as

$$
u^{*}(x)=-R^{-1} B^{T} \sum_{n=0}^{\infty} \quad \mu^{n} \frac{\partial \bar{v}_{n}}{\partial x} \quad(x)
$$

observe the difference between the results obtained above and that obtained by Garrard [13].

The Method of Solution of First-order Linear Partial Differential Equations The general solution of a first-order partial differential equation can be obtained analytically using the method of characteristics [9]. This method reduces the partial differential equation to a problem in ordinary differential equations. These first-order partial differential equations can also be solved numerically by finite-element methods [18].

Conclusions: A systematic perturbation method has been developed based on the Bellman's Fundamental Technique of perturbation. The method can be applied to solve a class of nonlinear analytic systems whose dependent variable (solution variables) can be expanded in a regular perturbation series near the nominal solutions. The salient feature of the method is 
that the auxiliary equation used in the Technique is nonlinear. Thus the central point of the success of the method lies in choosing a suitable auxiliary equation (whose solution is known) which should be as close in form to the nonlinear problem to be solved as possible. 
REFERENCES

1. IEEE Trans. on Automatic Control, Vol. 19, No. 6, December 1974.

2. M. Athans and P. Falb, Optimal Control, New York, McGraw-Hill, 1966

3. B.D.O. Anderson and J. B. Moore, Linear Optimal Control, Prentice Hall, New Jersey, 1971.

4. H. Kwakernaak and R. Sivan, Linear Optimal Control of Systems, Wiley Interscience, New York, 1972.

5. R. Bellman and R. Bucy, "Asymptotic Control Theory", SIAM J. Control, Vol, 2, No. 1. 1964, pp. 11-18.

6. E. G. Al'brekhat, "Optimal Stabilization of Nonlinear Systems", J. Appl. Math. of Mich. (25), 1961, pp. 1254-1266.

7. R. E. Kalman, "Contributions to the Theory of Optimal Control", Bol. Soc. Motem. Mex.1960, pp. 102-109.

8. J. P. Dauer, "Nonlinear Perturbations of Quasi-linear Control Systems", J. Math. Anal. and Appl., 54, pp. 717-725 (1976) .

9. R. Courant and D. Hilbert, Methods of Mathematical Physics Vol II, Wiley (Interscience, New York, 1962.

10. R. Bellman, Perturbation Techniques in Mathematics, Physics and Engineering, Holt, Rinehart \& Winston, New York, 1964.

11. V. Volterra, Theory of Functionals and Integral and Integradifferential Equations, Dover Publication, 1959.

12. W. I. Garrard, N. H. McClamroch and L. G. Clark, "An Approach to SubOptimal Feedback Control of Nonlinear Systems", Int. J. Control, Vol. 10, No. 6, pp. 657-663 (1969).

13. W. L. Garrard, "Sub Optimal Feedback Control for Nonlinear Systems", Automatica, Vol. 8, pp. 219-221, 1972.

14. J. F. Baldwin and J. H. Sims Williams, "The Use of a Method of Perturbation in the Synthesis of Closed-loop Optimal Control Laws for Nonlinear Systems", Automatica (5), pp. 357-367 (1969).

15. N. Nishikawa, N. Sannomiya, and H. Itakwa, "A Method for Sub-optimal Design of Nonlinear Feedback Systems", Automatica, Vol. 7, pp. 703-712, 1971. 
16. D. L. Lukes, "Optimal Regulation of Nonlinear Dynamical Systems", SIAM J. Control, Vol. 7, No. 1, pp. 75-100, February 1969.

17. E. B. Lee and L. Markus, Foundations of Optimal Control Theory, John Wiley, New York, 1967.

18. G. Strang and G. Fix, Analysis of Finite-Element Methods, Prentice Hall, N.J., 1973. 